\title{
A TWO-DIMENSIONAL MODEL FOR LARGE-SCALE BUSHFIRE SPREAD
}

\author{
LAWRENCE K. FORBES ${ }^{1}$
}

(Received 10 July 1996; revised 10 July 1996)

\begin{abstract}
A model for bushfire spread is proposed, in which radiative heat transfer, species consumption and flammable gas production are taken into account. It is shown that fire propagation in this model does not occur as a one-dimensional travelling wave, except for smouldering combustion of wet bushland. Numerical solutions for the evolution of a line fire are obtained using a diagonally implicit finite difference scheme, and the effects of firebreaks and uncleared combustible debris are studied. An energy theorem is presented for the case of a spreading two-dimensional fire, and numerical results are illustrated.
\end{abstract}

\section{Introduction}

In some countries of the world, violent forest fires occur reasonably regularly, and the time interval between them is governed by the speed of regeneration of the trees. Perhaps the most famous are the fires that occur in the hills surrounding the city of Los Angeles.

In Australia, the population centres in the south-east corner of the country are not infrequently at risk from large-scale bushfires. The size and ferocity of these fires often distinguish them from forest fires in other parts of the world, and there are several factors which contribute to the savagery of these occurrences.

Severe Australian bushfires usually occur towards the end of the summer months (December to February), and often follow an intense heat wave, during which daytime temperatures can exceed $40^{\circ} \mathrm{C}$. Another factor which adds to the likelihood of a bushfire is the presence of strong winds. In the southern half of Australia, the summertime weather systems come from the west, and move towards the east; consequently, the bushland in the south-east corner can be subject to days of hot, dry, westerly winds that have travelled across thousands of kilometres of desert. Under such conditions,

\footnotetext{
'Department of Mathematics, University of Queensland, St. Lucia, Queensland 4072, AUSTRALIA
} (C) Australian Mathematical Society, 1997, Serial-fee code 0334-2700/97 
the natural vegetation rapidly becomes tinder dry. A third factor contributing to the somewhat unique character of southern Australian bushfires is the nature of the trees themselves. The Australian forest consists of a combination of several species of eucalyptus tree, for which fire forms an essential ingredient in the breeding cycle.

In 1983, the southern states of Victoria and South Australia experienced an unusually destructive fire over thousands of hectares of bushland, farm country and rural population centres. This fire is now known as the "Ash Wednesday" fire after the day it started, and claimed about 70 lives. In January 1994, another enormous fire system engulfed the eastern reaches of the state of New South Wales. At the height of the blaze, a fire front consisting of over 120 separate fires stretched approximately 600 kilometres in a north-south line, and consumed about 600,000 hectares of bushland, along with 185 houses. This is an area greater than twice the size of the European country of Luxembourg, and bigger than the American state of Delaware. Television footage claimed that 30 metre high flame fronts were fanned by 70 kilometre per hour winds.

Several authors have attempted to model large-scale forest fires mathematically. The review article by Catchpole and de Mestre [3] surveys a number of the earlier models. Subsequently, there has been much interest in travelling-wave phenomena, analogous to the famous Fisher-Kolmogorov diffusion wave in biology (see, for example, Murray [8]). It will be argued in this paper that moving wave fronts of this type are really more appropriate to smouldering combustion in porous media, perhaps, than to the progress of fully developed bushfires.

Nevertheless, the travelling non-linear diffusion wave has a strong appeal, and is advocated by Weber [15], [16] and Tang and Weber [13] as a model deserving of further attention. Matkowsky and Sivashinsky [7] modelled solid fuel combustion using the travelling wave approach. A single non-linear parabolic equation (the FrankKamenetskii equation) expressing a balance between heat diffusion and exothermic production has been studied by Gray and Kordylewski [5] and Buonincontri and Hagstrom [2], and in both papers, the presence of travelling wave solutions was established. A system of two coupled non-linear reaction-diffusion equations expressing diffusion and production of heat and the consumption of a solid has been investigated by Tam [12], and the existence of travelling waves has again been proved.

Travelling wave solutions seem particularly appropriate in the case of slowly moving combustion fronts, such as occur in the smouldering of wet bushland, and this will be discussed more fully in Section 2. Norbury and Stuart [9] have investigated such waves in a porous medium, and show that there are two types of behaviour, depending upon whether the temperature at each end of the combustion wave falls below some ignition temperature, or else if there is complete exhaustion of the fuel upstream. A similar model, but one which is more complex, has been presented recently by Shkadinsky et al [10], and permits two-fronted travelling waves. A model in which 
the speed of the combustion wave is approximately constant is discussed for a similar system by Booty and Matkowsky [1]; in this case there is a gradual change to the shape of the wave front as it propagates.

Any serious attempt to model a fully developed bushfire is clearly fraught with great difficulty. In addition to the usual modelling uncertainties, such as the fact that the distribution of combustible fuel within a given forest is probably not uniform and may not even be known accurately, there are also more fundamental problems related to the understanding of the physical process itself. This point is emphasized in the review article of Weber [14]. In particular, a successful model should account for the fact that not every small fire develops into a large scale blaze. A relatively simple model of firespreading has been proposed by Fujii et al [4]; it treats the moving fire front as a Stefan problem in applied mathematics, but does not allow either for wind-driven effects or for fuel consumption. A later model of Grishin, Gruzin and Zverev [6] is more complete in this respect, but involves a difficult system of eight non-linear equations with ten subsidiary conditions.

In discussions with Australian fire-fighters, the view is often expressed that bushfires in eucalyptus forests can be aggravated by the presence of a volatile eucalyptus vapour, given off by the trees themselves in very hot weather. This would help to explain the sometimes extraordinary way in which fully developed bushfires can jump wide firebreaks; in the recent New South Wales fires, several television stations screened an amateur video recording that featured a fire front traversing a large river (the Hawkesbury river). In addition, the presence of intense "fire-balls" moving well ahead of the main fire front was described by many eye-witnesses, and possibly corresponds to pockets of burning gas that had been swept downstream.

Although much of this evidence is anecdotal, it surely cannot be dismissed lightly, and any useful model of a bushfire ought then to account for fiammable gas production. In this simple model, a solid fuel $S$ is therefore envisaged to decompose under heat in a dual reaction pathway, to form two different gaseous intermediates $G_{1}$ and $G_{2}$. These then burn to produce inert combustion products $P_{1}$ and $P_{2}$. The chemical scheme is thus



in which the quantities $k_{1}, \ldots, k_{4}$ are rate parameters. The first reaction pathway is intended to represent the rapid burning of the trees, while the second scheme is intended 
to allow for the production of the flammable gaseous substance $G_{2}$ corresponding to eucalyptus vapour. In each pathway, the first reactions (producing substances $G_{1}$ and $G_{2}$ ) are endothermic, and the second reactions (with rate parameters $k_{3}$ and $k_{4}$ ) are exothermic.

The direction and strength of prevailing winds are crucial factors in fire-fighting, and so it is necessary to incorporate the effects of a wind velocity vector $\mathbf{W}_{\mathrm{g}}$. The local rate equations for the substances $S, G_{1}$ and $G_{2}$ are then obtained from equations (1) according to first-order kinetics, and become

$$
\begin{aligned}
\frac{\partial S}{\partial t} & =-k_{1} S-k_{2} S, \\
\frac{D G_{1}}{D t} & =k_{1} S-k_{3} G_{1}, \\
\frac{D G_{2}}{D t} & =k_{2} S-k_{4} G_{2},
\end{aligned}
$$

where the derivative following the motion is defined in the usual way to be

$$
\frac{D G_{j}}{D t}=\frac{\partial G_{j}}{\partial t}+\mathbf{W}_{\mathrm{g}} \cdot \nabla G_{j} \quad j=1,2
$$

and the symbol $\nabla$ denotes the gradient operator. In (2) and (3), the names of the chemical species $S, G_{1}$ and $G_{2}$ have also been used to denote the concentrations of these substances.

It is, of course, the case that a more complete model of bushfire spread should also involve equations of motion for the gaseous products. However, the experience of fire-fighters is that prevailing winds are a primary factor in fire spread, so that when the winds die down, it is usually possible to bring the fire under control. For this reason, complicated second-order fluid mechanical effects are ignored in this simple model, which is concerned only with the prevailing wind velocity vector $\mathbf{W}_{\mathbf{g}}$.

As the present model is concerned with fire spread over a very large expanse of bushland, it follows that the horizontal length scales involved are several orders of magnitude greater than the vertical scale of the height of the flame front. Consequently, the variations of temperature in the vertical coordinate are represented only crudely here, by assuming separate temperatures $T_{s}$ and $T_{g}$ for the solid and gaseous phases, respectively.

The heat generated by a large bush fire can be extreme, and in the January 1994 bushfires near Sydney, for example, television footage showed that large steel supporting beams for buildings had been severely buckled, and thick glass windows melted. In such a temperature range, the rate parameters $k_{1}, \ldots, k_{4}$ will not be constants, but instead are strongly influenced by temperature. For the high temperature burning of the gaseous intermediates $G_{1}$ and $G_{2}$ it is reasonable to assume

$$
k_{j}=Z_{j} \exp \left(-E_{j} /\left(R T_{g}\right)\right) \quad j=3,4
$$


according to the usual Arrhenius law. Here, $E_{3}$ and $E_{4}$ are activation energies for the gases, and $R$ is the universal gas constant. The reference rates $Z_{3}$ and $Z_{4}$ are constants, and $T_{g}$ denotes the temperature in the gas.

Rate functions of the form (4) are perhaps not entirely appropriate for the parameters $k_{1}$ and $k_{2}$, however, since such a choice would lead to a type of "cold boundary" problem, with the solid fuel (bushland) being consumed for all temperatures above absolute zero. This unphysical effect would admittedly be exponentially small far from the fire, but since it would involve a very large area of bushland, it could nevertheless lead to unacceptably large energy losses, of spurious origin, in the model. To overcome this difficulty, the rate functions $k_{1}$ and $k_{2}$ are assumed to have the "shifted" Arrhenius forms

$$
k_{j}=\left\{\begin{array}{ll}
0 & \text { if } T_{s}<T_{j}^{I} \\
Z_{j} \exp \left(-1 /\left(T_{s} / T_{j}^{\prime}-1\right)\right) & \text { if } T_{s}>T_{j}^{I}
\end{array} \quad j=1,2 .\right.
$$

Here, the constants $T_{1}^{I}$ and $T_{2}^{\prime}$ are effectively ignition temperatures for the burning of the solid fuel $S$ according to the reaction pathways (1), following the approach of Norbury and Stuart [9]. The use of rate functions (5) that become exactly zero below some given temperature is not strictly necessary in this model, but the temperatures $T_{j}^{l}, j=1,2$ might possibly retain significance in terms of accounting for the complex internal chemistry of living organic matter, such as a tree, as it begins to ignite. A more complete model might replace this approximation (5) with a small-scale representation of these effects.

The assumption of temperature dependent rate parameters $k_{1}, \ldots, k_{4}$ in (4) and (5) requires appropriate energy balances in both the solid and the gas. If $\rho_{s}$ and $c_{s}$ denote the density and heat capacity of the solid fuel, then it follows from the assumed chemistry (1) that

$$
\rho_{s} c_{s} \frac{\partial T_{s}}{\partial t}=4 K_{R s} \nabla \cdot\left(T_{s}^{3} \nabla T_{s}\right)+K_{C s} \nabla^{2} T_{s}-h\left(T_{s}-T_{g}\right)-H_{1} k_{1} S-H_{2} k_{2} S .
$$

The first term on the right-hand side of this equation is an approximate representation of the effects of radiation. In (6), the constants $K_{R s}$ and $K_{C s}$ are respectively the coefficients of radiative and conductive heat transfer in the solid, $h$ is the heat transfer rate from the solid to the gaseous phase, and $H_{1}$ and $H_{2}$ are the heating coefficients for the production of gases $G_{1}$ and $G_{2}$ in the scheme (1); their negative sign indicates that these reactions are endothermic. There is a similar energy equation for the gas phase, namely,

$$
\rho_{g} c_{g} \frac{D T_{g}}{D t}=4 K_{R_{g}} \nabla \cdot\left(T_{g}^{3} \nabla T_{g}\right)-h\left(T_{g}-T_{s}\right)+H_{3} k_{3} G_{1}+H_{4} k_{4} G_{2},
$$


where the positive signs of the terms involving the heat release coefficients $\mathrm{H}_{3}$ and $\mathrm{H}_{4}$ indicate that these gaseous phase reactions are exothermic. The material derivative on the left of this equation is as previously defined in (3).

In order to keep this prototype bushfire model as simple as possible, the number of governing partial differential equations is now reduced by one, using a simple approximation. It is supposed that the burning of the gaseous intermediate $G_{1}$ to form product $P_{1}$, in the scheme (1), occurs over a much smaller time scale than any of the other processes. On the time scale of interest, the gas $G_{1}$ is therefore in a state of quasi-equilibrium with solid $S$. From the second equation in the scheme (2), there results the approximation

$$
G_{1}=\left(k_{1} / k_{3}\right) S
$$

and terms involving $G_{1}$ are now eliminated from the remaining partial differential equations using this relation.

Non-dimensional variables are now introduced, in which the reference unit for time is the quantity $1 / Z_{1}$ (where the rate $Z_{1}$ is as defined in (5)), and some appropriate quantity $L_{R}$ is chosen as the length scale. The solid concentration $S$ is made dimensionless using the reference quantity $S_{R}=\rho_{s} c_{s} T_{1}^{l} / H_{1}$, and a non-dimensional variable $G=G_{2} / S_{R}$ is used to describe the concentration of the second gaseous intermediate $G_{2}$. In these new scaled variables, the temperature of the solid is denoted $u=T_{s} / T_{1}^{I}$ and the temperature in the gas phase is $v=T_{g} / T_{1}^{I}$.

In non-dimensional form, the governing equations (2)-(7), under the approximation (8), become

$$
\begin{aligned}
\mu\left(\frac{\partial v}{\partial t}+\mathbf{W}_{\mathbf{g}} \cdot \nabla v\right) & =4 \lambda_{g} \nabla \cdot\left(v^{3} \nabla v\right)-\ell(v-u)+\gamma_{3} S k_{1}(u)+\gamma_{4} \kappa_{g} G k_{4}(v) \\
\frac{\partial u}{\partial t} & =4 \lambda_{s} \nabla \cdot\left(u^{3} \nabla u\right)+\sigma_{s} \nabla^{2} u-\ell(u-v)-S k_{1}(u)-\gamma_{2} \kappa_{s} S k_{2}(u) \\
\frac{\partial S}{\partial t} & =-S k_{1}(u)-\kappa_{s} S k_{2}(u) \\
\frac{\partial G}{\partial t}+\mathbf{W}_{\mathrm{g}} \cdot \nabla G & =\kappa_{s} S k_{2}(u)-\kappa_{g} G k_{4}(v)
\end{aligned}
$$

in which the dimensionless rate functions are defined to be

$$
\begin{aligned}
& k_{1}(u)=\left\{\begin{array}{ll}
\exp (-1 /(u-1)) & \text { if } u>1 \\
0 & \text { if } u<1
\end{array},\right. \\
& k_{2}(u)= \begin{cases}\exp \left(-\epsilon_{s} /\left(u-\epsilon_{s}\right)\right) & \text { if } u>\epsilon_{s} \\
0 & \text { if } u<\epsilon_{s}\end{cases}
\end{aligned}
$$


and

$$
k_{4}(v)=\exp \left(-\epsilon_{g} / v\right)
$$

Solutions to the model (9)-(10) are dependent upon the values of twelve dimensionless parameters. These are the ratio of thermal capacities $\mu=\left(\rho_{g} c_{g}\right) /\left(\rho_{s} c_{s}\right)$ and the radiation coefficients in the gaseous and solid phases

$$
\lambda_{g}=\frac{K_{R g}\left(T_{1}^{l}\right)^{3}}{\rho_{s} c_{s} Z_{1} L_{R}^{2}} \quad \text { and } \quad \lambda_{s}=\frac{K_{R s}\left(T_{1}^{l}\right)^{3}}{\rho_{s} c_{s} Z_{1} L_{R}^{2}}
$$

respectively. There is also the heat conduction coefficient $\sigma_{s}=K_{C s} /\left(\rho_{s} c_{s} Z_{1} L_{R}^{2}\right)$ within the solid, and the transfer rate $\ell=h /\left(\rho_{s} c_{s} Z_{1}\right)$ between the solid and gas phases. The constants $\epsilon_{s}=T_{2}^{l} / T_{1}^{\prime}$ and $\epsilon_{g}=E_{4} / R T_{1}^{\prime}$ are essentially activation temperatures for the burning of the solid and the gas, respectively, while $\kappa_{s}=Z_{2} / Z_{1}$ and $\kappa_{g}=Z_{4} / Z_{1}$ measure the rates at which these reactions occur. The second stage of the reaction (1) has endothermic heat coefficient $\gamma_{2}=H_{2} / H_{1}$ and the exothermic reactions in each stage occur with relative heat release coefficients $\gamma_{3}=H_{3} / H_{1}$ and $\gamma_{4}=H_{4} / H_{1}$.

This model (9)-(10) is still relatively simple, yet manages to account approximately for the effects of wind, radiative and conductive heat transfer, flammable gas production above a certain temperature, and an elementary treatment of the chemistry of combustion. Furthermore, it is considerably simpler than the model of Grishin, Gruzin and Zverev [6], and is amenable to analysis. Nevertheless, a large number of dimensionless parameters is required, and as Weber [16] points out, these can be very difficult to estimate on the basis of field or laboratory measurements. It is the purpose of this paper, however, to show that the description of a large bushfire requires a model of at least comparable complexity to the present one, which is capable of exhibiting many of the qualitative features of a spreading fire.

\section{One-dimensional travelling wave solutions}

It is the purpose of this section to examine the possibility that a line fire (in one dimension) might move as a travelling wave, similar to those studied by Norbury and Stuart [9]. It will be argued here that a large bushfire does not generally progress in this fashion.

The central result of this section is the following theorem.

THEOREM 1. A necessary condition for the propagation of a one-dimensional travel- 
ling wave in the model (9) - (10) is that

$$
\frac{\gamma_{3}-1}{\gamma_{2}-\gamma_{4}}>0
$$

Proof. Suppose that the wind velocity vector is $\mathbf{W}_{\mathrm{g}}=U_{g} \mathbf{i}$, and the wave front has position $x=\theta(t)$ at time $t$. The travelling wave solution takes the form

$$
v(x, t) \equiv v(\eta), \quad \eta=x-\theta(t),
$$

with similar expressions for the other dependent variables $u, S$ and $G$. Substituting the expressions (12) into (9), in one dimension only, then gives rise to the system of four coupled ordinary differential equations

$$
\begin{aligned}
\mu\left(U_{g}-\dot{\theta}\right) \frac{d v}{d \eta} & =4 \lambda_{g} \frac{d}{d \eta}\left(v^{3} \frac{d v}{d \eta}\right)-\ell(v-u)+\gamma_{3} S k_{1}(u)+\gamma_{4} \kappa_{g} G k_{4}(v) \\
-\dot{\theta} \frac{d u}{d \eta} & =4 \lambda_{s} \frac{d}{d \eta}\left(u^{3} \frac{d u}{d \eta}\right)+\sigma_{s} \frac{d^{2} u}{d \eta^{2}}-\ell(u-v)-S k_{1}(u)-\gamma_{2} \kappa_{s} S k_{2}(u) \\
-\dot{\theta} \frac{d S}{d \eta} & =-S k_{1}(u)-\kappa_{s} S k_{2}(u) \\
\left(U_{g}-\dot{\theta}\right) \frac{d G}{d \eta} & =\kappa_{s} S k_{2}(u)-\kappa_{g} G k_{4}(v)
\end{aligned}
$$

The boundary conditions at each end of the wave are that $u(\eta), v(\eta) \rightarrow u_{a}$ and $u^{\prime}(\eta), v^{\prime}(\eta) \rightarrow 0$ as $\eta \rightarrow \pm \infty$, where $u_{a}$ denotes the ambient temperature. (The values of the solid concentration $S$ may differ far upstream and far downstream of the wave.) Equations (13) are integrated, and the first, second and fourth are combined to give the result

$$
\kappa_{s} \int_{-\infty}^{\infty} S k_{2}(u) d \eta=\left(\frac{\gamma_{3}-1}{\gamma_{2}-\gamma_{4}}\right) \int_{-\infty}^{\infty} S k_{1}(u) d \eta .
$$

Since the integrands on both sides of the (14) must be positive, it follows that $\left(\gamma_{3}-\right.$ 1) $/\left(\gamma_{2}-\gamma_{4}\right)>0$ for a wave to propagate. This confirms condition (11), and concludes the proof of Theorem 1 .

The inequality (11) can be satisfied in one of two ways. If $\gamma_{3}>1$, so that the first reaction scheme (with product $P_{1}$ ) in (1) is overall exothermic, then it must follow that $\gamma_{2}>\gamma_{4}$. In this case, the second reaction pathway in (1) consumes more heat than it produces. Conversely, if $\gamma_{3}<1$ then also it is necessary that $\gamma_{2}<\gamma_{4}$; although the second pathway (with product $P_{2}$ ) is now nett exothermic, the first reaction scheme has now become overall endothermic. 
Thus it follows that, at least in the present model, a travelling wave in one dimension is only sustainable if one of the two reaction schemes in (1) is endothermic overall. This is scarcely likely to be the case under the conditions of extreme drying that characterize large bushfires. Consequently, fully developed bushfires are unlikely to propagate as simple travelling waves.

It is, of course, the case that the inequality (11) could be satisfied by wet bushland, for which one of the reaction pathways in (1) was nett endothermic, representing the fact that the fire front first had to drive off water vapour from the wet forest before it could burn. A model of this type is considered by Sisson et al [11], and concerns the smouldering combustion of damp cellulosic materials (such as bagasse or wood shavings).

\section{Numerical solution for a line fire}

This section briefly reviews the diagonally implicit finite-difference scheme developed for the solution of this problem in one dimension. A spatial mesh $x_{j}$, $j=1,2, \ldots, N$ is introduced, along with a difference grid $t_{k}, k=1,2, \ldots, P$ for the time. The dependent variables $u\left(x_{j}, t_{k}\right)$, and so on, are approximated by point values $u_{j}^{k}$ on the numerical grid, and it is convenient to introduce the central difference operators

$$
\begin{aligned}
& \delta_{x}^{1} u_{j}^{k}=u_{j+1}^{k}-u_{j-1}^{k}, \\
& \delta_{x}^{2} u_{j}^{k}=u_{j+1}^{k}-2 u_{j}^{k}+u_{j-1}^{k} .
\end{aligned}
$$

In one spatial dimension, with the wind velocity vector expressed in the form $\mathbf{W}_{\mathrm{g}}=U_{g} \mathbf{i}$, the system of equations (9) is approximated by the difference formulae

$$
\begin{gathered}
\mu\left(\frac{v_{j}^{k+1}-v_{j}^{k}}{\Delta t}+\frac{1}{2} U_{g} \frac{\delta_{x}^{1} v_{j}^{k}+\delta_{x}^{1} v_{j}^{k+1}}{2 \Delta x}\right) \\
=12 \lambda_{g}\left(v_{j}^{k}\right)^{2}\left(\frac{\delta_{x}^{1} v_{j}^{k}}{2 \Delta x}\right)^{2}+2 \lambda_{g}\left(v_{j}^{k}\right)^{3} \frac{\delta_{x}^{2} v_{j}^{k}+\delta_{x}^{2} v_{j}^{k+1}}{\Delta x^{2}} \\
\quad-\ell\left(\frac{1}{2}\left[v_{j}^{k}+v_{j}^{k+1}\right]-u_{j}^{k}\right)+\gamma_{3} S_{j}^{k} k_{1}\left(u_{j}^{k}\right)+\gamma_{4} \kappa_{g} G_{j}^{k} k_{4}\left(v_{j}^{k}\right), \\
\frac{u_{j}^{k+1}-u_{j}^{k}}{\Delta t}=12 \lambda_{s}\left(u_{j}^{k}\right)^{2}\left(\frac{\delta_{x}^{1} u_{j}^{k}}{2 \Delta x}\right)^{2}+\frac{1}{2}\left[\sigma_{s}+4 \lambda_{s}\left(u_{j}^{k}\right)^{3}\right] \frac{\delta_{x}^{2} u_{j}^{k}+\delta_{x}^{2} u_{j}^{k+1}}{\Delta x^{2}}, \\
\left.\frac{S_{j}^{k+1}-S_{j}^{k}}{\Delta t}=-\ell\left(\frac{1}{2}\left[u_{j}^{k}+u_{j}^{k+1}\right]-v_{j}^{k}\right)-S_{j}^{k} k_{1}\left(u_{j}^{k}\right)-\gamma_{2} \kappa_{s} S_{j}^{k} k_{2}\left(u_{j}^{k}\right)+\kappa_{s}^{k} k_{2}\left(u_{j}^{k}\right)\right]\left(S_{j}^{k}+S_{j}^{k+1}\right),
\end{gathered}
$$




$$
\frac{G_{j}^{k+1}-G_{j}^{k}}{\Delta t}+\frac{1}{2} U_{g} \frac{\delta_{x}^{1} G_{j}^{k}+\delta_{x}^{1} G_{j}^{k+1}}{2 \Delta x}=\kappa_{s} S_{j}^{k} k_{2}\left(u_{j}^{k}\right)-\frac{1}{2} \kappa_{g} k_{4}\left(v_{j}^{k}\right)\left[G_{j}^{k}+G_{j}^{k+1}\right] .
$$

It is easy to show, using a von Neumann stability analysis, that a difference scheme of this type would be unconditionally stable for a linear, constant-coefficient system of equations similar to (9), and this consideration has guided the development of the finite-difference scheme (16). Nevertheless, non-linearity has an adverse effect on the stability of (16), and in practice, it is found that the numerical time step $\Delta t$ must usually be chosen to be of order $\Delta x^{2}$.

To close the system, the difference scheme (16) is solved together with the Neumann boundary conditions

$$
\frac{\partial v}{\partial x}=\frac{\partial u}{\partial x}=\frac{\partial G}{\partial x}=0
$$

at each end of the computational window, $x=x_{1}$ and $x=x_{N}$. The conditions (17) are approximated using the centred difference operator $\delta_{x}^{1}$ in (15), and values of the dependent variables at the "false boundary" grid points $x_{0}=x_{1}-\Delta x$ and $x_{N+1}=x_{N}+\Delta x$ are eliminated using the difference formulae (16) applied at the boundary, in the usual way.

The numerical solution is thus advanced from the $k$-th time level to the $(k+1)$ st level by solving a tri-diagonal matrix system for each one of the four dependent variables $v, u, S$ and $G$. In view of the fact that the closure conditions (17) actually represent insulated boundaries, the calculation must stop before the moving fire front reaches the edges of the computational window.

\section{Numerical study of a moving line fire}

A great many numerical solutions has been computed, using the difference scheme of Section 3, and a good understanding of the behaviour of the model (9)-(10) has been developed for a one-dimensional fire. In particular, the fact that the present model does not automatically predict a fire growing in intensity is regarded as one of its considerable successes. For most choices of the parameters, it is observed that a fire established at time $t=0$ burns a little of the fuel and spreads slightly, before the solid temperature $u$ falls below the ignition temperatures $u=1$ and $u=\epsilon_{s}$, and the fire simply goes out. This behaviour reflects the situation that exists in practice, where intense bushfires are a relatively infrequent event.

Figure 1 shows the typical growth of a bushfire, under the rather rare conditions in which such growth is possible in this model. As Weber [16] has indicated, it is difficult to determine experimentally the parameter values that should be used, although in this 




(a)



(b)

FIGURE 1. Growth of a one-dimensional line fire. The wind speed is $U_{g}=2$, the ambient temperature is $u_{a}=0.75$ and the initial solid concentration is $S_{a}=1$. Parameter values are as given in the text. The solution is shown at (a) time $t=0.2$ and (b) time $t=0.6$.

paper, the heat capacity ratio is assumed to be $\mu=0.01$. The radiation and diffusion coefficients are set at the values $\lambda_{g}=\lambda_{s}=\sigma_{s}=0.1$; this choice is somewhat arbitrary since the reference length $L_{R}$ used in the definition of these constants has not been specified. The other parameter values used in Figure 1 are $\ell=5, \epsilon_{g}=\epsilon_{s}=1$, $\kappa_{g}=\kappa_{s}=5, \gamma_{2}=1$ and $\gamma_{3}=\gamma_{4}=15$. The ambient temperature is taken to be $u_{a}=0.75$ and the initial fuel concentration is $S_{a}=1$. The initial conditions used in Figure 1 were

$$
v(x, 0)=u_{a} \quad S(x, 0)=S_{a} \quad \text { and } \quad G(x, 0)=0
$$

and

$$
u(x, 0)= \begin{cases}u_{a} & \text { if }|x|>x_{w} \\ u_{a}+\frac{1}{2}\left(u_{M}-u_{a}\right)\left[1+\cos \left(\pi x / x_{w}\right)\right] & \text { if }|x|<x_{w}\end{cases}
$$

with $u_{M}=2.5$ and $x_{w}=0.5$.

Both Figures 1(a) and 1(b) have been drawn to the same scales, so that the growth of the fire is easily visible. After time $t=0.2$, Figure 1(a) indicates that the initial maximum value for the solid temperature, $u_{M}=2.5$, has been substantially reduced to a new maximum of about $u=1.4$. This is clearly due to energy transfer from the solid to the gas, and the production of a small concentration of flammable gas through consumption of some of the solid $S$. For later times, $t>0.2$, the fire gains rapidly in intensity, so that by $t=0.6$, as shown in Figure 1(b), a substantial portion of the solid fuel $S$ has been burnt, resulting in severe high temperatures in both solid 
and gas, and a growing concentration of flammable gas. The numerical results are not reliable for much later times than this, since the fire front then reaches the edges of the computational window shown, and the boundary conditions (17) cease to be appropriate.

A wind of strength $U_{g}=2$ has been assumed in these results, and its effect on the progress of the fire can be seen clearly. There is a shift of the maximum in the gas-temperature profile $v$ downstream to about $x=1.2$, as is to be expected with the wind speed used here. In addition, the flammable gas concentration $G$ likewise has a secondary peak at about $x=1.2$. From these profiles, then, it is evident that the fire does not develop simply as a travelling wave, confirming Theorem 1 for the parameter values assumed here.

A pleasing feature of the present model is that an initial disturbance such as (18) generally does not grow as shown in Figure 1, consistently with physical reality. (This is a consequence of the first endothermic steps in the reaction scheme (1), and the ignition temperature assumptions (5).) In fact, considerable numerical experimentation was needed in order to find a parameter set that would give the fire growth shown in these diagrams. It is useful now to study the conditions under which a bushfire can develop in this model, since they appear to accord with actual experience.

Whether or not a fire develops in this model, in the destructive manner indicated in Figure 1, is mainly governed by the values of the activation temperatures $\epsilon_{g}$ and $\epsilon_{s}$, the rate constants $\kappa_{g}$ and $\kappa_{s}$ and the heat release coefficients $\gamma_{3}$ and $\gamma_{4}$. The model appears to be reasonably insensitive to changes in the other parameters.

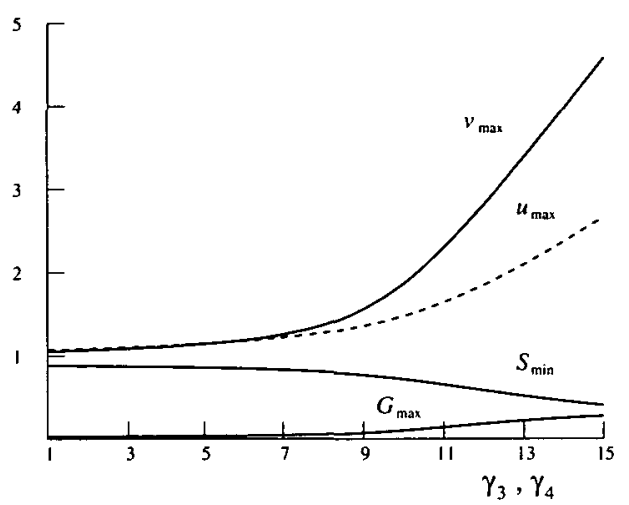

FIGURE 2. The dependence of maximum gas and solid temperatures $v$ and $u$, minimum solid concentration $S$ and maximum flammable gas concentration $G$ upon the thermal release coefficients $\gamma_{3}=\gamma_{4}$, at time $t=0.6$. Here, $\epsilon_{g}=\epsilon_{s}=1$ and $\kappa_{g}=\kappa_{s}=5$. The other parameters and initial condition (18) are given in the text.

In Figure 2, the extremum values of the two temperatures $u$ and $v$, the solid fuel concentration $S$ and the flammable gas concentration $G$ are plotted as functions of 
the heat release coefficients, at time $t=0.6$. In this diagram, the two ignition temperatures are fixed at the value $\epsilon_{g}=\epsilon_{s}=1$ and the two rate coefficients are maintained as $\kappa_{g}=\kappa_{s}=5$. For simplicity, the two heat release rates $\gamma_{3}$ and $\gamma_{4}$ have been made equal in this figure; similar, if less spectacular, results are obtained under conditions of differing heat releases, and so the choice $\gamma_{3}=\gamma_{4}$ is primarily for ease of viewing.

If the heat release coefficients $\gamma_{3}$ and $\gamma_{4}$ are sufficiently small, then it is clear from Figure 2 that the fire does not grow. Instead, a small amount of the fuel $S$ is consumed, and the fire spreads a little before going out. Our numerical results indicate that the fire apparently does not grow significantly until the heat release rates are about $\gamma_{3}=\gamma_{4}=9$, assuming the same initial conditions as in (18).

A similar threshold effect for bushfire development is observed in Figure 3, in which the extremum values of the functions $u, v, S$ and $G$ are shown, as they vary with the rate constants $\kappa_{g}$ and $\kappa_{s}$ (again assumed equal, for ease of presentation). Here, the activation temperatures are $\epsilon_{g}=\epsilon_{s}=1$, and the heat release coefficients are now maintained at the value $\gamma_{3}=\gamma_{4}=15$. The initial conditions are as given in (18), and results are shown for time $t=0.6$. If the chemical rate coefficients $\kappa_{g}$ and $\kappa_{s}$ are suitably small, it is evident that the fire cannot grow in intensity, and the numerical results suggest that a fully developed fire is only possible for rate constants $\kappa_{g}$ and $\kappa_{s}$ slightly in excess of about 2 .

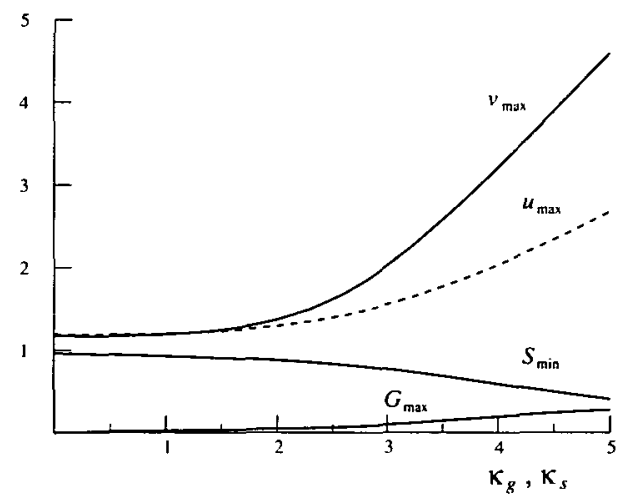

FIGURE 3. The dependence of maximum gas and solid temperatures $v$ and $u$, minimum solid concentration $S$ and maximum flammable gas concentration $G$ upon the rate constants $\kappa_{g}=\kappa_{s}$, at time $t=0.6$. Here, $\epsilon_{8}=\epsilon_{s}=1$ and $\gamma_{3}=\gamma_{4}=15$.

In summary, it is therefore the case that conditions which are likely to lead to the growth of a large fire are a low value of activation energy $\epsilon_{g}$ and ignition temperature $\epsilon_{s}$, high rate constants $\kappa_{g}$ and $\kappa_{s}$ and high heat release constants $\gamma_{3}$ and $\gamma_{4}$. If these conditions are not met, the fire loses intensity and goes out. This observation 
is consistent with the anecdotal wisdom of firefighters, since the prolonged hot dry weather that normally precedes an intense Australian bushfire would be sufficient to bring about all these effects, essentially by means of the severe drying that takes place in such conditions.

Once a large scale bushfire has established itself, it is usually not possible to fight it directly, in view of the intense heat generated and the high winds that often accompany it. Instead, the usual management strategy is to cut firebreaks; these are regions such as paddocks or roads where the fuel available to the oncoming fire is scarce. Often, firefighters will engage in "backburning", in an attempt to remove even more of the fuel between the chosen firebreak and the advancing fire front.

This use of firebreaks as a containment strategy for a bushfire has been studied with the present model, and a typical result is shown in Figure 4. A wind of strength $U_{g}=2$ in the positive $x$-direction is again present, and all other conditions are as in Figure 1, except that now the initial function for the distribution of solid fuel has been taken to be

$$
S(x, 0)= \begin{cases}0 & \text { if } 1<x<3 \\ 1 & \text { otherwise }\end{cases}
$$

Profiles for the gas and solid temperatures, $v$ and $u$, and the solid concentration $S$ and flammable gas concentration $G$ are again shown at time $t=0.6$, so that a direct comparison with Figure $1(b)$ is possible.

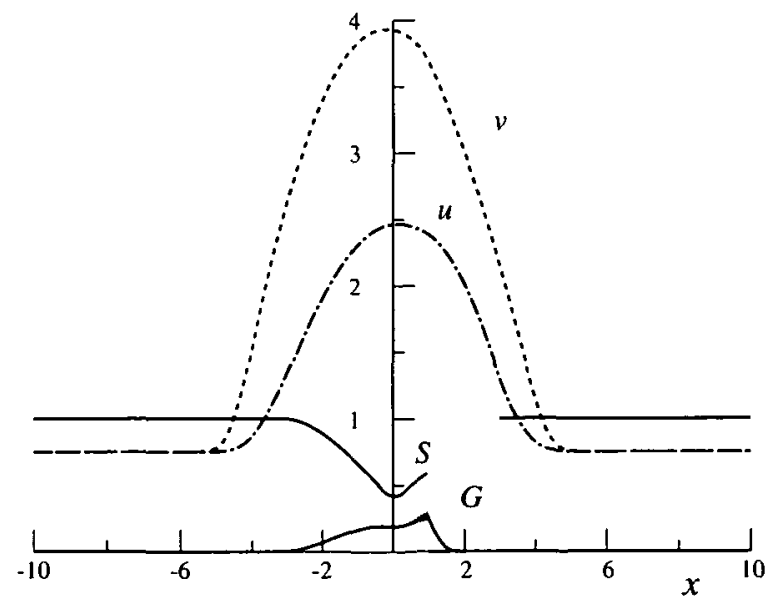

FIGURE 4. The effect of a firebreak, created using initial conditions $S=0$ in $1<x<3$, and $S=1$ otherwise. The parameters values are as in Figure 1.

It is at once clear from Figure 4 that the presence of the firebreak at $1<x<3$ has had a significant effect on the progress of the fire. The most noticeable effect is that 
a substantial reduction in the temperatures $v$ and $u$ of the gas and solid, respectively, has occurred, even in the portions of the fire before the firebreak. There is much less of the flammable gas $G$ present in the fire. For times greater than $t=0.6$, some consumption of the fuel beyond the firebreak does, in fact, take place; thus the firebreak is eventually breached due to radiation effects from the hot air convected downstream by the wind. This also occurs in the field, and it is standard practice to patrol the downstream side of a firebreak, putting out small spot fires when they are detected.

In the aftermath of the 1994 bushfires in New South Wales, there has been much concern about the role played by uncleared forest debris in regions near houses and suburbs. There is no doubt that bushfires are aided by the presence of such debris, and this has also been studied with the present model.

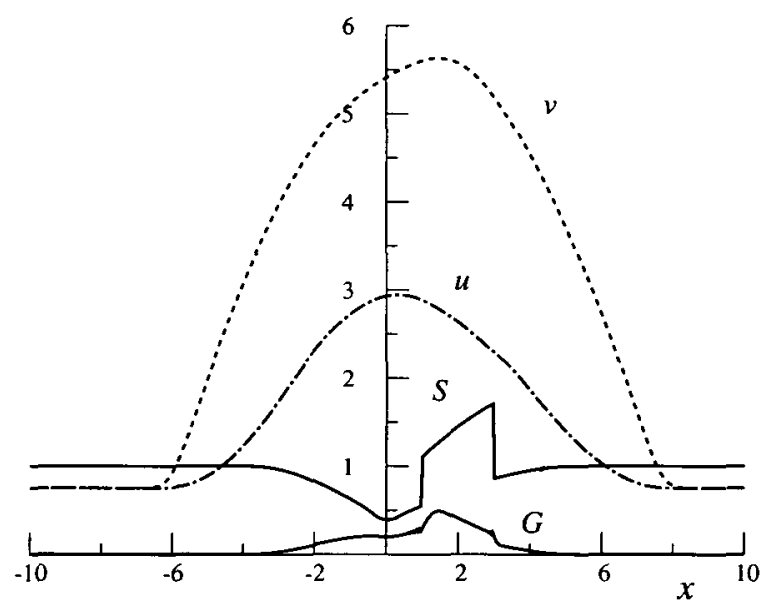

FIGURE 5. The effect of excess fuel, created using initial conditions $S=2$ in $1<x<3$, and $S=1$ otherwise. The parameters values are as in Figure 1.

In Figure 5, profiles are shown at time $t=0.6$ for the same conditions as in Figures 1 and 4, except that the initial profile (19) has been replaced with

$$
S(x, 0)= \begin{cases}2 & \text { if } 1<x<3 \\ 1 & \text { otherwise }\end{cases}
$$

as a simple model of a region $1<x<3$ of uncleared debris. The effect on the development of the fire is at once apparent. The fire front extends further downstream and the temperature $v$ of the air, in particular, is very greatly increased. In this diagram, as in Figure 4, the small oscillations in the gas concentration profile $G$ are a consequence of the discontinuous initial conditions (19) and (20). They are an 
artifice of the numerical solution only (through the Gibbs' phenomenon), and are of no consequence.

\section{Spread of a planar fire, and its numerical computation}

From the perspective of a firefighter, it is probably the case that the one-dimensional (large scale) line fire is of most practical interest. Nevertheless, it is possible also to consider the initial stages of fire development with this model (although the vertical structure of the flame, ignored here, may be of greater significance in this case).

THEOREM 2. If the gaseous phase is assumed incompressible, then there is a suffciently small value of the parameter $\gamma_{2}$ for which the total thermal energy of the fire will increase, provided that $\gamma_{3}>1$.

PROOF. In dimensional variables, the thermal energy per unit volume is $\rho_{s} c_{s} T_{s}+\rho_{g} c_{g} T_{g}$ and so it is appropriate to consider the total dimensionless thermal energy

$$
E=\iiint(u+\mu v) d V
$$

The boundary conditions are that ambient conditions prevail far away, so that $u, v \rightarrow$ $u_{a}$ and $G \rightarrow 0$ as $x^{2}+y^{2} \rightarrow \infty$.

The four partial differential equations (9) are integrated over the entire volume. By the divergence theorem, it follows that

$$
\iiint \nabla \cdot\left(u^{3} \nabla u\right) d V=\oint u^{3} \frac{\partial u}{\partial n} d S=0,
$$

since $u$ is constant at infinity. Thus the integrals of the radiation terms involving $u$ and $v$ both vanish, and a similar argument applies to the conduction term (with coefficient $\sigma_{s}$ ) in the solid phase, also.

If the gaseous phase is incompressible, then $\operatorname{div} \mathbf{W}_{\mathrm{g}}=0$, and the integral of the convective term in the first equation in (9) may be written

$$
\iiint \mathbf{W}_{\mathbf{g}} \cdot \nabla v d V=\iiint \operatorname{div}\left(v \mathbf{W}_{\mathrm{g}}\right) d V=u_{a} \oint \mathbf{W}_{\mathbf{g}} \cdot \mathbf{n} d S .
$$

Here, the divergence theorem and boundary conditions at infinity have been used. Applying the divergence theorem again to (23) gives

$$
\iiint \mathbf{W}_{\mathrm{g}} \cdot \nabla v d V=u_{a} \iiint \operatorname{div} \mathbf{W}_{\mathrm{g}} d V=0
$$


by virtue of the incompressibility of the gas. A zero result for the integral of the convective term in the last equation in the system (9) may be derived in the same fashion as (23) and (24), using the boundary condition $G \rightarrow 0$ at infinity.

After a little algebra, the integrated equations (9) may be combined to yield

$$
\begin{aligned}
\frac{d E}{d t}= & \left(\gamma_{3}-1\right) \iiint S k_{1}(u) d V \\
& +\left(\gamma_{4}-\gamma_{2}\right) \kappa_{g} \iiint G k_{4}(v) d V-\gamma_{2} \frac{d}{d t} \iiint G d V .
\end{aligned}
$$

If $\gamma_{3}>1$, then the first term on the right-hand side is positive. It follows that if $\gamma_{2}$ is sufficiently small, the entire right-hand side of (25) can be guaranteed positive, in which case the derivative $d E / d t$ of the energy defined in equation (21) is positive. Thus the energy increases with time, and the theorem is proved.

The results of Theorem 2 can be understood without difficulty in terms of the physics of the situation. If $\gamma_{3}>1$ and $\gamma_{2}$ is appropriately small, then the chemical reactions (1) are exothermic overall, and the total thermal energy in the fire would be expected to increase.

A numerical method has been devised for the solution of (9) in a planar region. It is similar to the diagonally implicit finite-difference scheme used in Section 3, but in addition makes use of an alternating-direction implicit (ADI) methodology. As before, a spatial mesh $\left(x_{i}, y_{j}\right), i=1,2, \ldots, N, j=1,2, \ldots, M$ is defined, along with a temporal grid $t_{k}, k=1,2, \ldots, P$.

There is insufficient space here to write out the difference formulae for each of the four equations in (9), as in (16), so the procedure will be illustrated in reference to the first equation in this system, with obvious generalization to the other three. The difference operators (15) are now extended to two dimensions, in the form

$$
\begin{aligned}
\delta_{x}^{1} u_{i, j}^{k}=u_{i+1, j}^{k}-u_{i-1, j}^{k}, & \delta_{y}^{1} u_{i, j}^{k}=u_{i, j+1}^{k}-u_{i, j-1}^{k}, \\
\delta_{x}^{2} u_{i, j}^{k}=u_{i+1, j}^{k}-2 u_{i, j}^{k}+u_{i-1, j}^{k}, & \delta_{y}^{2} u_{i, j}^{k}=u_{i, j+1}^{k}-2 u_{i, j}^{k}+u_{i, j-1}^{k} .
\end{aligned}
$$

The wind velocity vector is assumed to have the cartesian form $\mathbf{W}_{\mathrm{g}}=U_{g} \mathbf{i}+V_{g} \mathbf{j}$.

In the first phase of the solution method, the dependent variables are found at the half time-step $t_{k+1 / 2}$. For the first equation in the system (9), the difference 
approximation is

$$
\begin{gathered}
\mu\left[\frac{v_{i, j}^{k+1 / 2}-v_{i, j}^{k}}{\Delta t / 2}+U_{g} \frac{\delta_{x}^{1} v_{i, j}^{k+1 / 2}}{2 \Delta x}+V_{g} \frac{\delta_{y}^{1} v_{i, j}^{k}}{2 \Delta y}\right] \\
=4 \lambda_{g}\left(v_{i, j}^{k}\right)^{3}\left[\frac{\delta_{x}^{2} v_{i, j}^{k+1 / 2}}{\Delta x^{2}}+\frac{\delta_{y}^{2} v_{i, j}^{k}}{\Delta y^{2}}\right]+12 \lambda_{g}\left(v_{i, j}^{k}\right)^{2}\left[\left(\frac{\delta_{x}^{1} v_{i, j}^{k}}{2 \Delta x}\right)^{2}+\left(\frac{\delta_{y}^{1} v_{i, j}^{k}}{2 \Delta y}\right)^{2}\right] \\
-\ell\left(v_{i, j}^{k+1 / 2}-u_{i, j}^{k}\right)+\gamma_{3} S_{i, j}^{k} k_{1}\left(u_{i, j}^{k}\right)+\gamma_{4} \kappa_{g} G_{i, j}^{k} k_{4}\left(v_{i, j}^{k}\right),
\end{gathered}
$$

and this can be solved as a tri-diagonal matrix system for the gas temperature $v_{i, j}^{k+1 / 2}$, $i=1,2, \ldots, N$ for each value of the index $j$. As in Section 3, Neumann boundary conditions of the type (17) have been assumed, with "false boundaries" beyond the computational window as before. Similar diagonally-implicit difference schemes are obtained for the other dependent variables in the system (9).

The second phase of the method advances the solution from time level $t_{k+1 / 2}$ to the next full time level $t_{k+1}$, as required, by means of the difference formula

$$
\begin{aligned}
& \mu\left[\frac{v_{i, j}^{k+1}-v_{i, j}^{k+1 / 2}}{\Delta t / 2}+U_{g} \frac{\delta_{x}^{1} v_{i, j}^{k+1 / 2}}{2 \Delta x}+V_{g} \frac{\delta_{y}^{1} v_{i, j}^{k+1}}{2 \Delta y}\right] \\
& =4 \lambda_{g}\left(v_{i, j}^{k+1 / 2}\right)^{3}\left[\frac{\delta_{x}^{2} v_{i, j}^{k+1 / 2}}{\Delta x^{2}}+\frac{\delta_{y}^{2} v_{i, j}^{k+1}}{\Delta y^{2}}\right] \\
& \quad+12 \lambda_{g}\left(v_{i, j}^{k+1 / 2}\right)^{2}\left[\left(\frac{\delta_{x}^{1} v_{i, j}^{k+1 / 2}}{2 \Delta x}\right)^{2}+\left(\frac{\delta_{y}^{1} v_{i, j}^{k+1 / 2}}{2 \Delta y}\right)^{2}\right] \\
& \quad-\ell\left(v_{i, j}^{k+1}-u_{i, j}^{k+1 / 2}\right)+\gamma_{3} S_{i, j}^{k+1 / 2} k_{1}\left(u_{i, j}^{k+1 / 2}\right)+\gamma_{4} \kappa_{g} G_{i, j}^{k+1 / 2} k_{4}\left(v_{i, j}^{k+1 / 2}\right) .
\end{aligned}
$$

The solution $v_{i, j}^{k+1}, j=1,2, \ldots, M$ is again obtained as the solution to a tri-diagonal system, for each value of index $i$, with Neumann conditions (17) assumed at the boundaries of the computational window. This ADI semi-implicit finite-difference scheme (27)-(28) can readily be shown to have unconditional numerical stability for linear, constant-coefficient partial differential equations similar to (9), although in application it is again found that the non-linearity of the system (9) degrades this stability characteristic, as for the one-dimensional solution method outlined in Section 3.

\section{Preliminary numerical results for a planar fire}

A reasonably large number of numerical simulations has been performed using the scheme outlined in Section 5, for spreading planar fires. Some preliminary attempts 
to investigate the effects of different starting conditions, variable fuel loading and wind effects have been undertaken, but the full range of possibilities here clearly go well beyond the scope of this investigation. Accordingly, an indicative sample only of results that can be obtained with this model will be presented in this section.

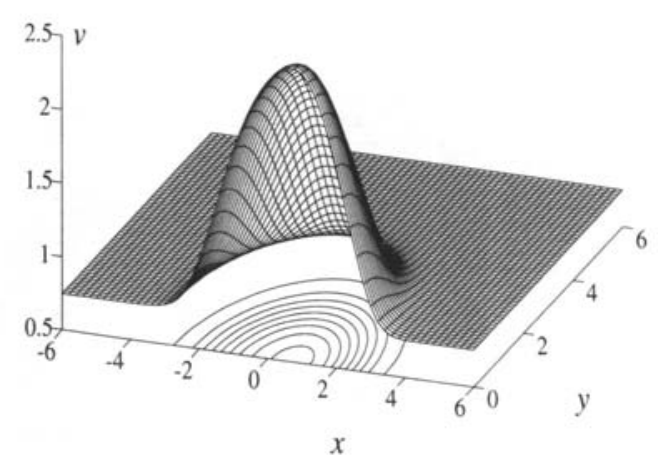

FIGURE 6. Development of a planar fire from the initial condition (29). The profile shown is for the gas temperature $v(x, y)$ at time $t=0.6$.

Figure 6 shows the gas-temperature profile $v$ at time $t=0.6$, for the same parameter values as used in Figure 1, and an initial condition

$$
u(x, y, 0)= \begin{cases}u_{a} & \text { if } x^{2}+y^{2}>r_{w}^{2} \\ u_{a}+\frac{1}{2}\left(u_{M}-u_{a}\right)\left[1+\cos \left(\pi \sqrt{x^{2}+y^{2}} / r_{w}\right)\right] & \text { if } x^{2}+y^{2}<r_{w}^{2},\end{cases}
$$

with $u_{M}=2.5$ and $r_{w}=1.2$. The variables $v, S$ and $G$ have initial values given by (18a), and a wind vector $W_{g}=2 i$ is present. In this example, the initial fire clearly gains in intensity and spreads as time is increased. In addition, there is noticeable distortion of the plume towards the positive $x$-direction, as a consequence of the wind.

Contour maps are presented for this case, for the solid and gas temperatures $u$ and $v$ and the concentrations of solid $S$ and flammable gas $G$, in Figures 7(a)-(d) respectively. These profiles show that the effect of the wind is to distort the initially circular plume into a more elliptical pattern by time $t=0.6$. In addition, the location of the maximum in the gas-temperature profile $v$ has moved significantly downstream, as expected.

A large number of simulations has been performed for the spreading planar fire, but the possibilities are too extensive to be discussed at length here. To conclude this 


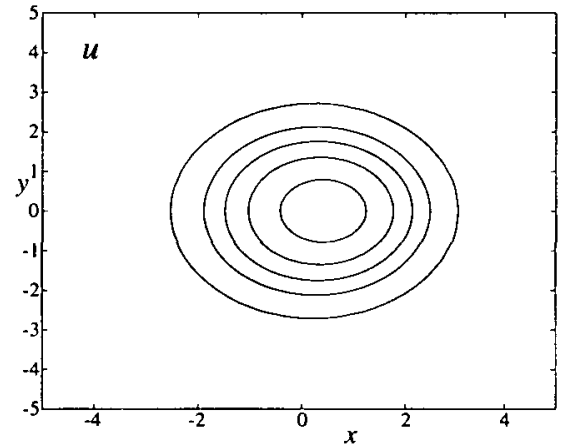

(a)

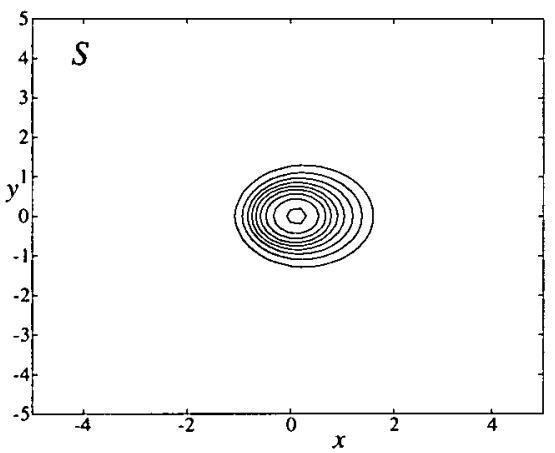

(c)

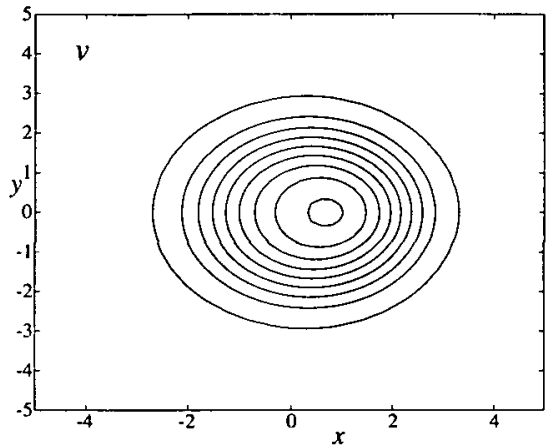

(b)

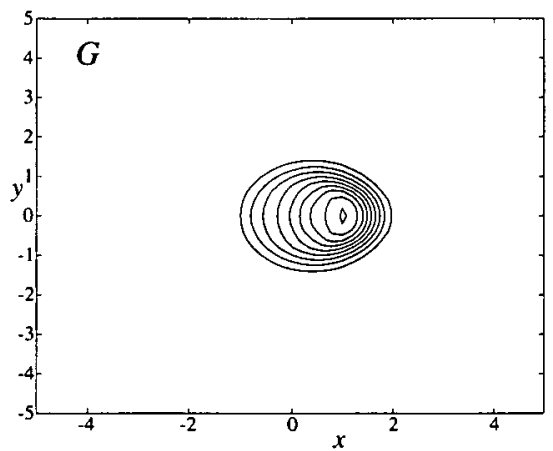

(d)

FiguRE 7. Contour maps for the planar fire in Figure 6 at time $t=0.6$. Results are shown for (a) the solid temperature $u$, (b) gas temperature $v$, (c) solid concentration $S$ and (d) gas concentration $G$. 
section, the effects of extra fuel on the fire are studied under the same conditions as before, except that the initial condition for the solid concentration $S$ is now altered to

$$
S(x, y, 0)= \begin{cases}S_{a} & \text { if } r_{1}>1.2 \\ S_{a}+\frac{1}{2}\left(S_{M}-S_{a}\right)\left[1+\cos \left(\pi r_{1} / 1.2\right)\right] & \text { if } r_{1}<1.2,\end{cases}
$$

in which $r_{1}^{2}=(x-2.4)^{2}+y^{2}$ and the maximum initial concentration is $S_{M}=2 S_{a}$, with $S_{a}=1$ as before.



FIGURE 8. Solution profile and contour map for the gas temperature $v$ at time $t=0.6$, for a planar fire with initial conditions (29) and (30).

The gas-temperature profile $v$ and the solid concentration $S$ are shown at time $t=0.6$ for this case, in Figures 8 and 9 respectively. Here, the assumed symmetry of the profile about the plane $y=0$ was utilized in order to reduce the overall computer run time, and results are therefore only shown for $y>0$. A contour map is also included in each diagram, and Figure 9, in particular, shows the way in which the burn pattern is beginning to form a transverse crescent shape, in response to the wind and the extra fuel.

\section{Concluding remarks}

A simple mathematical model (9) has been proposed for the spread of a bushfire, and takes account of radiative effects, fuel consumption and wind. Its predictions appear to be in accordance with expectation and empirical fact. In particular, it has the feature that many parameter conditions do not result in a growing fire at all, but instead allow the fire simply to die out. Thus the model offers an explanation of why small 


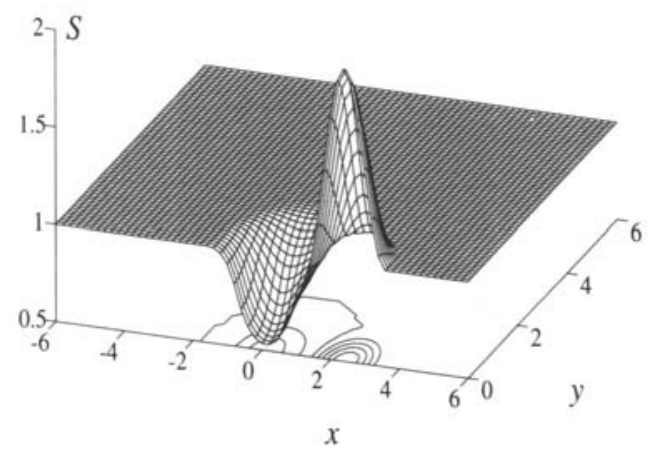

FIGURE 9. Solution profile and contour map for the solid concentration $S$ at time $t=0.6$, for a planar fire with initial conditions (29) and (30).

changes in ambient temperature (relative to absolute zero) can make the difference between gentle smouldering and a large scale bushfire. The answer suggested here is that it is the drying that occurs with high ambient temperatures and dry winds that ultimately creates conditions conducive to fire development, through an increase in the overall exothermicity of the chemical reactions involved in combustion, and a decrease in the activation energy for burning to occur.

These conclusions are very much in accordance with practical experience in the field, and for fire-fighters such knowledge has long been part of established understanding. It is appropriate therefore to conclude this paper with an assessment of what has been achieved by a model such as this, and what gains might be made in the future.

The present model has evidently provided a qualitatively reasonable account of the mechanisms responsible for fire spread; in particular, it has shown that fully developed bushfires are unlikely to propagate as mathematical travelling waves. It is possible that this model could be useful in enabling practitioners to decide how wide a firebreak needs to be, in order to cope with a particular distribution of solid fuel, for example.

In this relatively simple model, the vertical structure of the fire has not been addressed in detail, but has been modelled crudely by the use of two different temperatures $u$ and $v$. The justification for this is that vertical scales are some orders of magnitude less than horizontal ones, so that the progress of a genuinely large scale bushfire is unlikely to be greatly affected by the correct modelling of this third dimension. Nevertheless, there may be merit in improving the model with a more rigorous treatment of the vertical fire structure, particularly when examining the initial stages of two-dimensional fire spread. 
A worthwhile improvement to the present model (9) would also appear to be possible, by taking explicit account of the moisture content of the fuel. This could be done by allowing some dependence of the crucial parameters $\gamma_{3}, \gamma_{4}$ and $\epsilon_{g}$, for example, upon this moisture content. Some useful predictive capability of the likelihood of a destructive bushfire might well come from an improvement of this type. Finally, a model such as that proposed here, when used in conjunction with supercomputing capabilities and a database of forestry information, might at last enable the path of a large bushfire to be predicted well in advance, if all the empirical constants can be determined.

\section{Acknowledgements}

I am grateful to Mr. Harry C. Morris (Adelaide), an airconditioning engineer and a volunteer fire-fighter with Country Fire Service units in the Adelaide Hills. His keen observations and astute recollections formed the basis for much of the modelling in this paper. Material from the "Adelaide Advertiser" and the "Australian" newspapers, on the tenth anniversary of the 1983 Ash Wednesday fires, has proved useful, as have newspaper and television reports on the 1994 New South Wales bushfires. Some reference material was kindly supplied by Dr. R. Weber (ADFA, Canberra). All computations were performed on a 486 personal computer, purchased under ARC grant A691939.

\section{References}

[1] M. R. Booty and B. J. Matkowsky, "Slowly varying filtration combustion waves", Euro. J. Appl. Math. 4 (1993) 205-224.

[2] S. Buonincontri and T. Hagstrom, "Multidimensional travelling wave solutions to reactiondiffusion equations", IMA J. Appl. Maths. 43 (1989) 261-271.

[3] T. Catchpole and N. de Mestre, "Physical models for a spreading line fire", Aust. For. 49 (1986) 102-111.

[4] N. Fujii, J. Hasegawa, L. Pallop and Y. Sakawa, "A nonstationary model of firespreading", Appl. Math. Modelling 4 (1980) 176-180.

[5] P. Gray and W. Kordylewski, "Travelling waves in exothermic systems", Proc. Roy. Soc. London Ser. A 416 (1988) 103-113.

[6] A. M. Grishin, A. D. Gruzin and V. G. Zverev, "Mathematical modeling of the spreading of high-level forest fires", Sov. Phys. Dokl. 28 (1983) 328-330.

[7] B. J. Matkowsky and G. I. Sivashinsky, "Propagation of a pulsating reaction front in solid fuel combustion", SIAM J. Appl. Math. 39 (1979) 465-478.

[8] J. D. Murray, Mathematical Biology, (Biomathematics Texts Volume 19, Springer- Verlag, Berlin, 1989).

[9] J. Norbury and A. M. Stuart, "Travelling combustion waves in a porous medium. Part I - Existence", SIAM J. Appl. Math. 48 (1988) 155-169. 
[10] K. G. Shkadinsky, G. V. Shkadinskaya, B. J. Matkowsky and V. A. Volpert, "Two- front traveling waves in filtration combustion", SIAM J. Appl. Math. 53 (1993) 128-140.

[11] R. A. Sisson, A. Swift, G. C. Wake and B. F. Gray, "The self-heating of damp cellulosic materials: 1. High thermal conductivity and diffusivity", IMA J. Appl. Maths. 49 (1992) 273-291.

[12] K. K. Tam, "Traveling wave solutions for a combustion problem", Stud. Appl. Maths. 81 (1989) 117-124.

[13] S. Tang and R. O. Weber, "Numerical study of Fisher's equation by a Petrov- Galerkin finite element method", J. Austral. Math. Soc. Ser. B 33 (1991) 27-38.

[14] R. O. Weber, "Modelling fire spread through fuel beds", Prog. Energy Combust. Sci. 17 (1991) $67-82$.

[15] R. O. Weber, "Toward a comprehensive wildfire spread model", Int. J. Wildland Fire 1 (1991) 245-248.

[16] R. O. Weber, "Fire spread as a moving boundary", in Proc. Centre Maths. and its Applic. (eds. R. S. Anderssen, J. M. Hill and A. K. Pani) 30 (1992) 57-67. 